\title{
EDTA Surface Capped Water-Dispersible ZnSe and ZnS:Mn Nanocrystals
}

\author{
Jae-Woog Lee, Sang-Min Lee, Young-Duk Huh, and Cheong-Soo Hwang* \\ Department of Chemistry, Center for Photofunctional Energy Materials (GRRC), Dankook University, Yongin-Si, \\ Gyonggi 448-701, Korea. *E-mail: cshwang@dankook.ac.kr \\ Received March 15, 2010, Accepted May 25, 2010
}

\begin{abstract}
ZnSe and ZnS:Mn nanocrystals were synthesized via the thermal decomposition of their corresponding organometallic precursors in a hot coordinating solvent (TOP/TOPO) mixture. The organic surface capping agents were substituted with EDTA molecules to impart hydrophilic surface properties to the resulting nanocrystals. The optical properties of the water-dispersible nanocrystals were analyzed by UV-visible and room temperature solution photoluminescence (PL) spectroscopy. The powders were characterized by X-ray diffraction (XRD), high resolution transmission electron microscopy (HR-TEM), and confocal laser scanning microscopy (CLSM). The solution PL spectra revealed emission peaks at 390 (ZnSe-EDTA) and 597 (ZnS:Mn-EDTA) nm with PL efficiencies of 4.0 (former) and 2.4\% (latter), respectively. Two-photon spectra were obtained by fixing the excitation light source wavelengths at $616 \mathrm{~nm}$ (ZnSe-EDTA) and 560 $\mathrm{nm}$ (ZnS:Mn-EDTA). The emission peaks appeared at the same positions to that of the PL spectra but with lower peak intensity. In addition, the morphology and sizes of the nanocrystals were estimated from the corresponding HR-TEM images. The measured average particle sizes were $5.4 \mathrm{~nm}$ (ZnSe-EDTA) with a standard deviation of $1.2 \mathrm{~nm}$, and $4.7 \mathrm{~nm}$ (ZnS:Mn-EDTA) with a standard deviation of $0.8 \mathrm{~nm}$, respectively.
\end{abstract}

Key Words: ZnSe, ZnS:Mn, Water-dispersible nanoparticles, EDTA capping, Two-photon spectroscopy

\section{Introduction}

Nano-sized semiconducting materials have attracted considerable attention over the past decade. ${ }^{1,2}$ These materials have unique technological applications for various photo-electronic devices owing to their size dependent physical and optical properties. ${ }^{3-5}$ Recently developed nanocrystallite $\mathrm{ZnSe}$ is of particular interest on account of its intense UV-blue luminescent properties; an emission wavelength at $460 \mathrm{~nm}$ and a band gap of $2.7 \mathrm{eV}$, which are not observed in other nanocrystal materials, such as $\mathrm{CdS}, \mathrm{ZnS}$, and CdSe. ${ }^{6-8} \mathrm{In}$ addition, it was reported that when the surface of $\mathrm{ZnSe}$ is passivated by $\mathrm{ZnS}$, a core-shell type quantum dot is formed and the quantum yield is improved considerably compared to bare $\mathrm{ZnSe}$. $^{9,10}$

$\mathrm{ZnS}$ :Mn nanocrystals (an orange light emitting nanophosphor) have attracted substantial interest on account of their high photoluminescence efficiency and stability at ambient temperature, which are essential for commercial electro-luminescence devices. ${ }^{11}$ This study synthesized ZnS:Mn-EDTA nanocrystals and examined their unique optical and physical properties as a potential candidate for an optical probe in biomedical imaging. Previously, several bio-compatible amino acid molecule-capped $\mathrm{ZnS}: \mathrm{Mn}$ nanocrystals were synthesized in this laboratory. ${ }^{2,13}$

Water-dispersible semiconductor nanocrystals were developed especially for fluorescent labeling technologies in the biomedicinal research area. ${ }^{14,15}$ They are expected to replace complicated or hazardous radioactive detection, and have formed the basis of very sensitive biological assays. In addition, semiconductor nanocrystals are much more efficient, sensitive and stable than organic dyes used for the same purpose. Unfortunately, most highly luminescent semiconductor nanocrystals are grown in hydrophobic media, which makes them barely compatible with any biological system. There are several reports of solubilized hydrophobic nanocrystals in water. ${ }^{16-18}$ For example, the surface of $\mathrm{CdSe} / \mathrm{ZnS}$ semiconducting nanocrystal were coated with silica, mercaptoacetic acid or dihydolipoic acid in a buffer solution, and they were claimed to be waterdispersible semiconductor nanocrystals. ${ }^{19}$

This paper describes the synthesis of EDTA (ethylenediaminetetraacetic acid) surface capped water-dispersible $\mathrm{ZnSe}$ and $\mathrm{ZnS}: \mathrm{Mn}$ semiconductor nanocrystals along with their physical and optical properties. EDTA is a typical multidentate ligand in metal ion coordination chemistry, and is used frequently to mediate the morphology and size control for several bulk metal chalcogenide materials in hydrothermal synthetic methods. ${ }^{20-22}$ However, EDTA itself is rarely used as a surface capping agent for semiconductor nanocrystals. In this research, our primary goal of synthesizing the water-dispersible ZnSe-EDTA and ZnS:Mn-EDTA nanocrystals is to apply especially for bio-imaging and specific metal ion sensor area. EDTA molecule contains four carboxylate $\left(\mathrm{COO}^{-}\right)$arms to connect any peptide molecule via covalent bonding or to coordinate certain metal ions. In addition, the zinc ion containing nanocrystals are expected much less toxic than cadmium ion based nanocrystals such as CdSe and $\mathrm{CdS}$ to introduce directly into a living organism. Even though $\mathrm{ZnSe}$ and $\mathrm{ZnS}: \mathrm{Mn}$ nanocrystals have been mainly applied in electronic device area rather than bio-labeling technology due to their wide band gap, blue and orange emission wavelengths, we are trying to show that they can be also used as bioimaging agents in this article.

\section{Experimental Section}

Chemicals and instruments. All solvents used ( $n$-hexane, ethanol, and chloroform) were purchased from Aldrich and distilled prior to use. Diethylzinc (0.1 M solution in hexane), hexadecylamine (HDA, 90\%), tri- $n$-octylphosphine (TOP, 90\%), and selenium powder ( $\sim 100$ mesh $99.999 \%)$ were obtained from Aldrich and used as received. Hexamethyldisilathiane, (TMS) $)_{2} \mathrm{~S}$, was supplied by Fluka and used without further purification. 
A 1.0 M (TOP)Se stock solution was prepared in a dry box by mixing $0.78 \mathrm{~g}(0.01 \mathrm{~mol})$ of Se powder with the TOP solvent so that the total volume of the solution reached $10 \mathrm{~mL}$ in a volumetric flask. The UV-visible spectrum was recorded on a SCINCO 2001 spectrophotometer equipped with a deuterium/tungsten lamp. The solution FT-IR spectrum was recorded on a Bruker IRS-66/S spectrophotometer. The PL spectrum was obtained in a DARSA-2000 spectrophotometer equipped with a $500 \mathrm{~W}$ Xenon lamp, $0.275 \mathrm{~m}$ triple grating monochrometer, and a PHV 400 photomultiplitor tube. The PL efficiency of the corresponding ZnSe-EDTA and ZnS:Mn-EDTA were estimated by a comparison with the recommended standard material in the literature; ${ }^{23}$ a $0.1 \mathrm{M}$ aqueous solution of Tyrosine (Aldrich), whose excitation wavelength and reported absolute quantum yield are $275 \mathrm{~nm}$ and $14 \%$ at room temperature. The provided confocal laser scanning microscopy image was taken by OLYMPUS (OLS3000) equipped with $408 \mathrm{~nm}$ UV laser and halogen lamp as a light source. The powder X-ray diffraction (XRD, Rigaku 300) pattern was obtained using $\mathrm{Cu} \mathrm{K \alpha}(1.5418 \AA)$ radiation. The transmission electron microscopy (TEM, JEOL JEM 1210) images were taken in MAG mode of 1000 to 800000 and an accelerating voltage of $40-120 \mathrm{kV}$. Prior to analysis, the samples were dispersed in hexane and placed on a carbon-coated copper grid (300 Mesh) followed by drying in a vacuum. In addition, the elemental composition of the quantum dots was determined by energy dispersive X-ray spectroscopy (EDXS with a Si (Li) detector in IXRF 500 system) attached to the HR-TEM. In addition, an ICP-AES analysis for the ZnS:Mn-EDTA nanocrystal was performed by Optima-430 (Perkin Elmer) spectrometer equipped with Echelle optics system and Segmented array charge coupled device (SCD) detector. To prepare a sample of the ZnS:Mn-EDTA nanocrystal for ICP-AES analysis, a $0.5 \mathrm{~mL}$ of concentrated nanocrystal solution was mixed with $9.5 \mathrm{~mL}$ of concentrated nitric acid over the period of 3 days. After which $0.5 \mathrm{~mL}$ of the digested solution is placed in a $9.5 \mathrm{~mL}$ of nanopure-water. Finally, provided light scattering pictures of the nanocrystal containing colloids were taken by exposure of KIMMON IK3202-R He-Cd laser (325.0 nm) light.

Synthesis of ZnSe-EDTA nanocrystal. All manipulations were carried out using modified Schlenk techniques under an inert argon atmosphere. HDA $(15 \mathrm{~mL})$ was placed in a $200 \mathrm{~mL}$ three-neck flask connected to a vacuum manifold. The liquid was dried and degassed under vacuum at $c a .125^{\circ} \mathrm{C}$ for 7 hours. The flask was then filled with argon gas. A previously prepared $1 \mathrm{M}$ (TOP)Se stock solution $(0.6 \mathrm{~mL})$ was removed with a glass syringe from the dry box, and transferred immediately to a flask containing hot HDA solvent. A $0.1 \mathrm{M} \mathrm{Et}_{2} \mathrm{Zn}(6 \mathrm{~mL}, 0.6 \mathrm{mmol})$ in $n$-hexane solution was then added to the mixture solution at $300{ }^{\circ} \mathrm{C}$. After mixing the two precursors, a vigorous reaction occurred immediately, and the mixture was stirred for $30 \mathrm{minu}-$ tes. The mixture solution was cooled to $90{ }^{\circ} \mathrm{C}$ and incubated for 10 hours at this temperature. Further cooling to ambient temperature and the addition of anhydrous ethanol resulted in the formation of a white precipitate. The isolated $\mathrm{ZnSe}$ white powder, which was produced by multiple centrifuging and filtering, was redispersed in $25 \mathrm{~mL}$ of chloroform solvent. A $50 \mathrm{~mL}$ of a $0.1 \mathrm{M}$ solution of EDTA in water was placed in a separate flask. A $1 \mathrm{M}$ stock solution of $\mathrm{NaOH}$ was added until the $\mathrm{pH}$ reached 11. A $15 \mathrm{~mL}$ sample of the EDTA-NaOH mixed solution was transferred to a flask containing $\mathrm{ZnSe}$ nanocrystals. After stirring 3 hours, the aqueous layer was isolated using a separate funnel, and the nanocrystal product in the aqueous solution was characterized by UV-visible, Fourier transform infrared (FT-IR) and photoluminescence (PL) spectroscopy.

Synthesis of ZnS:Mn-EDTA nanocrystal. A mixture of 2.0 $\mathrm{mL}$ of $0.1 \mathrm{MEt}_{2} \mathrm{Zn}(2 \mathrm{mmol})$ and $0.42 \mathrm{~mL}$ of (TMS $)_{2} \mathrm{~S}$ (2 mmol) in TOP (tri- $n$-octylphosphine) was added dropwise to a flask containing bis(acetylacetonato)manganese (II) (0.01 g, 0.04 $\mathrm{mmol}$ ) in $25 \mathrm{~mL}$ of a TOP/TOPO (tri- $n$-octylphosphine oxide) solvent mixture at $135^{\circ} \mathrm{C}$. After stirring the mixture at this temperature for 30 minutes, the resulting solutions were cooled to ca. $90{ }^{\circ} \mathrm{C}$ and incubated for 10 hours. Off-white powders were obtained by adding anhydrous methanol to a flask containing the colloidal product at ambient temperature, which was followed by multiple centrifuging, filtering and vacuum drying. The substitution process of the TOPO molecules to EDTA for ZnS:Mn nanocrystal is identical to that for the ZnSe-EDTA nanocrystals. The separated aqueous solution was also characterized by UVvisible, FT-IR and PL spectroscopy. The PL efficiencies of the ZnSe-EDTA and ZnS: Mn-EDTA nanocrstals were measured and calculated using the method reported by Williams et al. ${ }^{24}$ This method involves calculating the relative quantum yield through a comparison with a standard material; a $0.1 \mathrm{M}$ aqueous solution of Tyrosine whose reported excitation wavelength and absolute quantum yield are $275 \mathrm{~nm}$ and $14 \%$, respectively. ${ }^{23}$ Table 1 gives a summary of the spectral and physical data of the corresponding nanocrystalline product.

\section{Discussions}

Figure 1 (a) and (b) present HR-TEM images of ZnSe-EDTA and $\mathrm{ZnS}: M n-E D T A$ nanocrystals. The measured average parti-

Table 1. Experimental Data Summary of ZnSe-EDTA and ZnS:Mn-EDTA nanocrystals

\begin{tabular}{lccc}
\hline & ZnSe-EDTA & ZnS:Mn-EDTA \\
\hline UV-vis $\left(\lambda_{\max }, \mathrm{nm}\right)$ & 308 & 280 \\
\hline PL emission wave lengths $(\mathrm{nm})$ & 390 & 4.0 & 570 \\
\hline PL efficiency $(\%)$ & $3296(\mathrm{~s}), 2970(\mathrm{w}), 1637(\mathrm{~s}), 1468(\mathrm{w})$, & $3299(\mathrm{~s}), 2118(\mathrm{~m}), 1634(\mathrm{~s}), 1404(\mathrm{w})$, \\
FT-IR $v\left(\mathrm{~cm}^{-1}\right)$ & $1394(\mathrm{w}), 1250(\mathrm{w}), 1057(\mathrm{w})$ & $1320(\mathrm{w}), 1136(\mathrm{w}), 1046(\mathrm{w})$ \\
HR-TEM image $($ average particle size, $\mathrm{nm})(\mathrm{RSD})$ & $5.4(1.2)$ & $4.7(0.8)$ \\
Lattice spacing $(\AA)$ & 3.3 & 3.6 \\
\hline
\end{tabular}


cle sizes were $5.4 \mathrm{~nm}$ (ZnSe-EDTA) with a standard deviation of $1.2 \mathrm{~nm}$, and $4.7 \mathrm{~nm}$ (ZnS:Mn-EDTA) with a standard deviation of $0.8 \mathrm{~nm}$, respectively. In addition, the appearance of distinct lattice planes in the fringe images with an approximate spacing of $3 \AA$ suggests that all the solid samples were made from single crystals rather than poly-crystalline aggregates. Energy dispersive X-ray spectra (EDXS) were obtained to confirm the elemental compositions of the ZnSe-EDTA and ZnS:Mn-EDTA nanocrystals in the solid state. The atomic ratios of $\mathrm{Zn}: \mathrm{Se}$ (ZnSeEDTA) and Zn:S (ZnS:Mn-EDTA) were almost 1:1, and the doping concentration of manganese (II) ions in the $\mathrm{ZnS}: \mathrm{Mn}-$ EDTA nanocrystal was approximately $0.7 \%$. The concentration of the manganese (II) ion in the ZnS:Mn-EDTA nanocrystal was precisely determined by an Inductively Coupled Plasma-Atomic Emission Spectrometry (ICP-AES) analysis. Three trials of the sample measurements provided that average elemental portion of the Mn ion against $\mathrm{ZnS}$ parent crystal was $1.33 \%$ (with $0.28 \%$ of RSD). The manganese (II) ion doping concentration in the $\mathrm{ZnS}$ : Mn crystals were originally aimed at approximately $1-2 \%$ because it was reported that the best PL efficiency was obtained in that doping range for other ligand-capped $\mathrm{ZnS}$ :Mn nanocrystals. ${ }^{25}$ Fig. 1 (c) and (d) present Scanning confocal fluorescence microscopy images for ZnSe-EDTA(c) and ZnS:MnEDTA (d) nanocrystals. The vast majority of the nanocrystals exist as single particles, which is demonstrated by well separated spots with sizes equal to the point spread function of the microscope. There were some aggregates in the picture and the small lumps of nanocrystal particles could be resulted from hydrogen bonding interactions between the EDTA molecules on the surface of the nanocrystals.

Fig. 2 (a) presents size histograms of ZnSe-EDTA and ZnS: Mn-EDTA nanocrystals. Both nanocrystals fairly fit into Gaus-
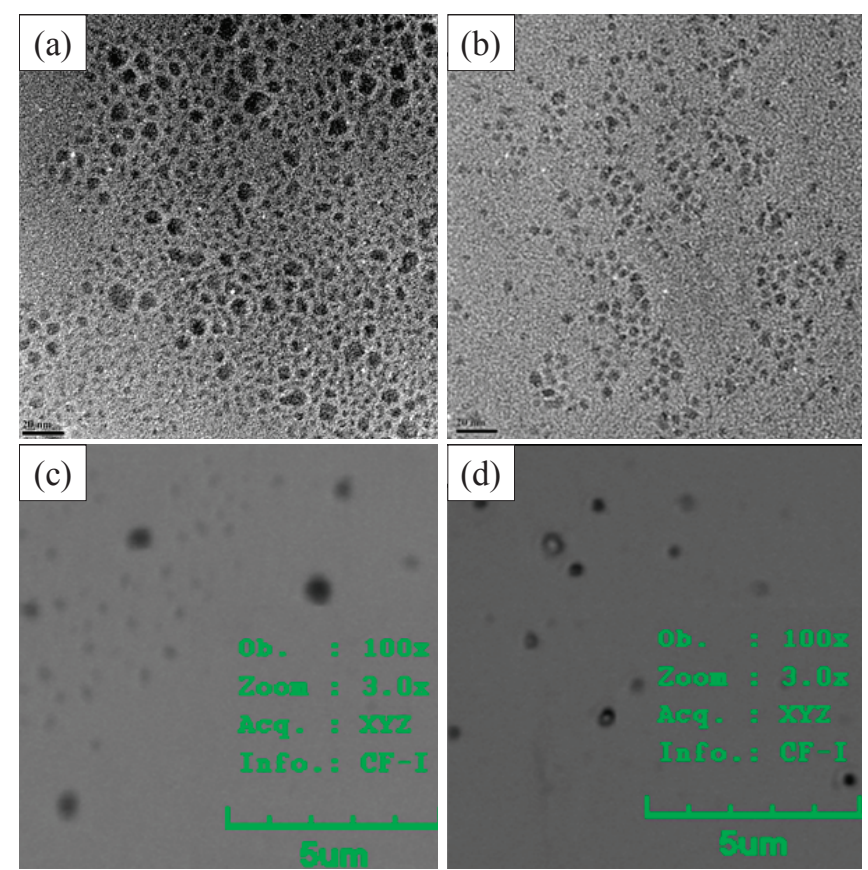

Figure 1. HR-TEM images of: (a) ZnSe-EDTA and (b) ZnS:Mn-EDTA, the scale bars represent $20 \mathrm{~nm}$, and Confocal scanning microscopy images of: (c) ZnSe-EDTA and (d) ZnS:Mn-EDTA. sian size distribution centered at $5.4 \mathrm{~nm}$ (ZnSe-EDTA) with a standard deviation of $1.2 \mathrm{~nm}$, and at $4.7 \mathrm{~nm}$ (ZnS:Mn-EDTA) with a standard deviation of $0.8 \mathrm{~nm}$. Fig. 2(b) and (c) pictures were taken by exposing $325 \mathrm{~nm} \mathrm{He}-\mathrm{Cd}$ laser light whose wavelength is close to the excitation wavelengths of the ZnSe-EDTA and $\mathrm{ZnS}$ :Mn-EDTA nanocrystals. The pictures show characteristic Tyndall light scatterings for colloidal solutions.

Figure 3 shows the photoluminescence (PL) spectroscopy data obtained from ZnSe-EDTA and ZnS:Mn-EDTA nanocrystals. The room temperature solution PL spectra, in Fig. 3(a) and (b), revealed broad emission peaks at 390 and $570 \mathrm{~nm}$, respectively. These emission spectra were obtained by fixing the excitation wavelengths of the light source at the corresponding UV-visible absorption wavelengths. From the spectra, both nanocrystals showed large Stokes shifts between the absorption and emission wavelengths. Usually the surface defect resulted from incomplete capping by the surfactant ligand or from metal or counter anion vacancy in the crystal lattice. For the $\mathrm{ZnSe}$ nanocrystal, the $c a .82 \mathrm{~nm}$ Stokes shift in the absorption-emission energy gap difference is described as a characteristic of quantum dot known to occur due to the presence of traps originating from surface levels and other intrinsic defects in literature. ${ }^{26}$ In some cases, weak orange light emission at $600 \mathrm{~nm}$ has been observed by Se ion vacancy in the crystal lattice, but we did not observe such peak in our ZnSe-EDTA nanocrystal sample. In addition, in the $\mathrm{ZnS}$ :Mn nanocrystal, the orange light emission at $570 \mathrm{~nm}$ is attributed to the ${ }^{4} \mathrm{~T}_{1-}{ }^{6} \mathrm{~A}_{1}$ transition of $\mathrm{Mn}^{2+}$ ions.
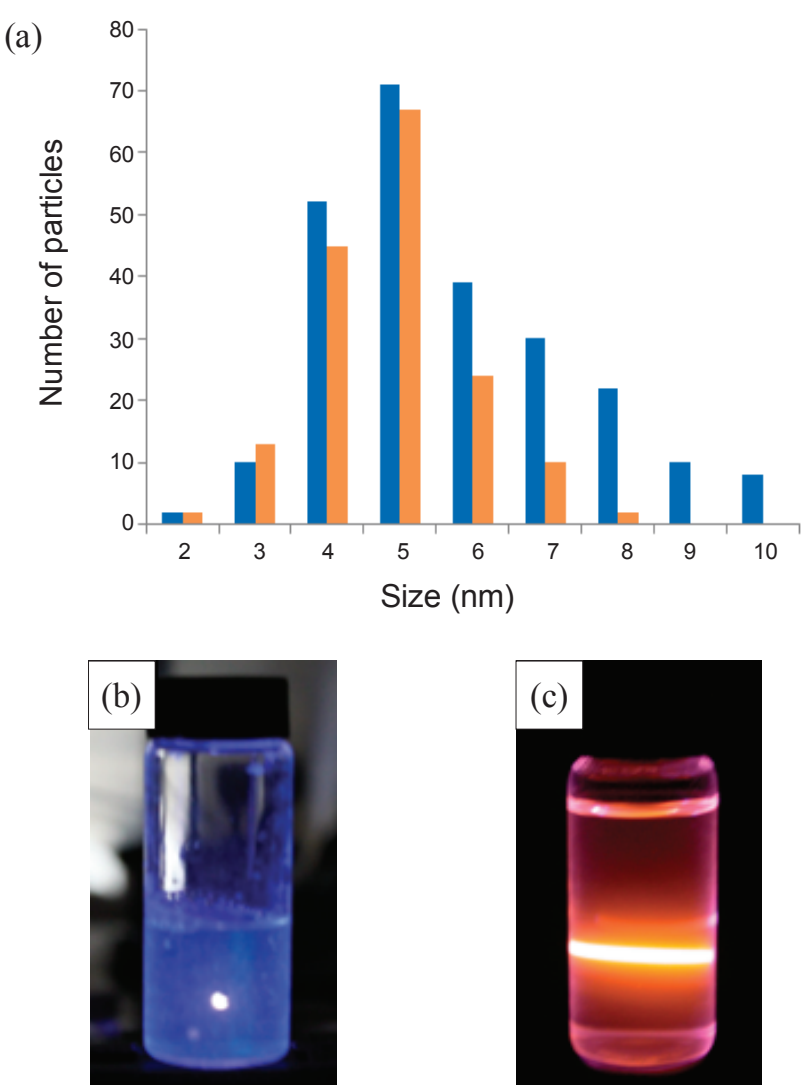

Figure 2. (a) Size histogram of ZnSe-EDTA (blue) and ZnS:Mn-EDTA (orange), and Light scattering pictures of (b) ZnSe-EDTA and (c) ZnS: Mn-EDTA nanocrystals exposed to He-Cd laser (325 nm) light. 
During the luminescence pathway, if surface defect states are located close to the conduction band, the direct energy transfer from the $\mathrm{ZnS}$ host to the $\mathrm{Mn}^{2+}$ dopant ion is significantly interrupted, which can cause weakening the orange emission as well as enlarging Stokes shift. ${ }^{27}$ In some cases, previously mentioned surface defect on the $\mathrm{ZnS}: \mathrm{Mn}$ nanocrystal and zinc ion vacancy can cause an extra blue emission around at $450 \mathrm{~nm}$, but we didn't observe such blue emission peak in our PL spectrum for the ZnS:Mn-EDTA nanocrystal. ${ }^{28}$

The calculated relative PL efficiencies of the nanocrystals were $4.0 \%$ (ZnSe-EDTA) and 2.4\% (ZnS:Mn-EDTA), which are much lower than those for nonpolar or organic ligands-capped $\mathrm{ZnSe}(15 \%)$ and $\mathrm{ZnS}: \mathrm{Mn}$ (5.1\%) nanocrystals. The PL efficiencies in aqueous solution for both nanocrystals decreased approximately same rate which one third from the organic environment. Luminescence quenching effect study for the various polar ligands capped $\mathrm{CdSe} / \mathrm{ZnS}$ quantum dot in aqueous solvent has been reported in the literature. ${ }^{29}$ According to the paper, molecules especially containing an acetate moiety such as mercaptoacetic acid showed much greater luminescence quenching effect than that having other terminal groups in aqueous solution. Quenching of molecular fluorescence by carbonyl group also has been explained to occur via electron transfer to its $\pi^{*}$ orbital. Therefore, it seems that surface defects associated with
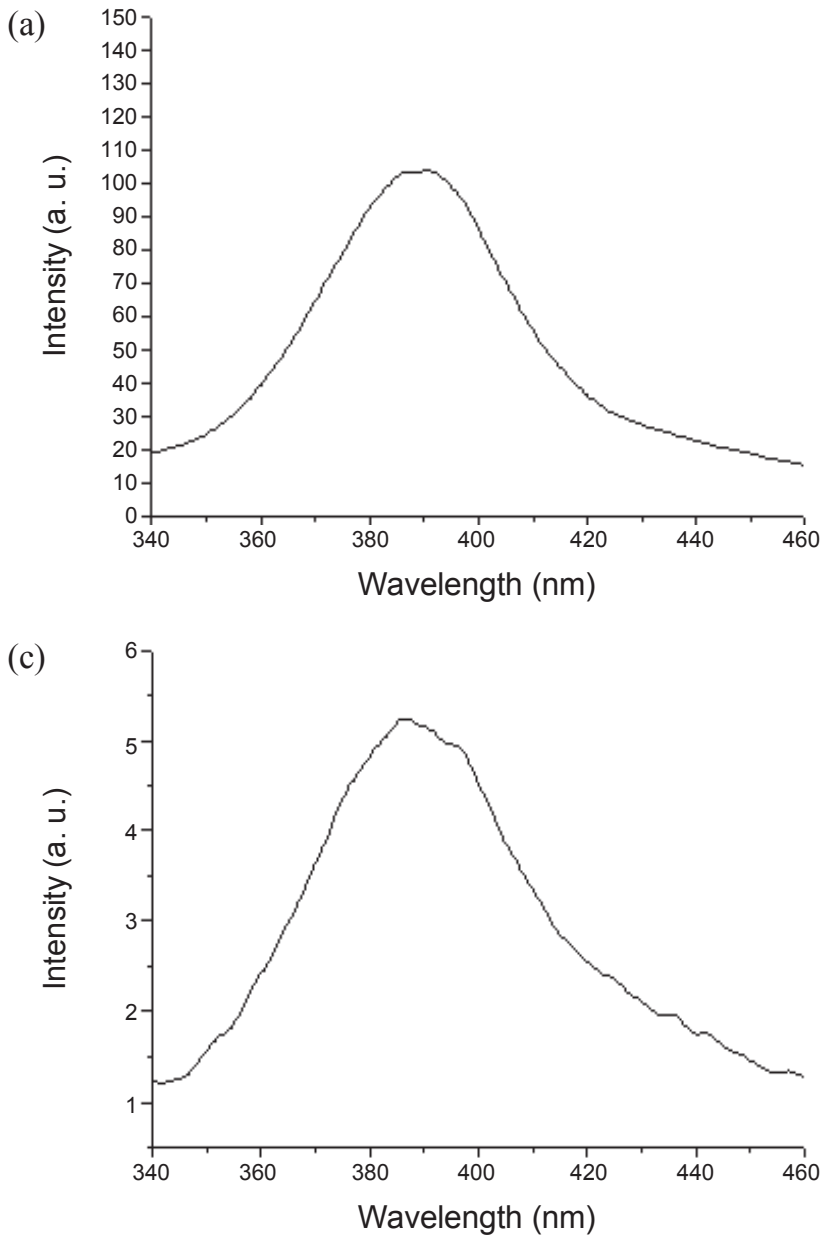

the presence of carbonyl groups in EDTA molecule enhance the luminescence quenching in the ZnSe-EDTA and ZnS:MnEDTA nanocrystals.

Figure 3 (c) and (d) show the two-photon spectra of $\mathrm{ZnSe}$ EDTA and ZnS:Mn-EDTA nanocrystals. Both spectra were obtained on a solution PL spectrophotometer by fixing the light source at the double wavelengths of the corresponding UVvisible absorption wavelengths for the nanocrystals. Although the peak intensities were significantly lower than that for the original PL emission peaks, the emission peaks could be identified at the same position with the corresponding original PL emission peaks. Identifiable two-photon emission peaks are especially significant for biological applications of nanocrystals. Scanning a biological cell with a high energy light source may not be appropriate because it might be harmful for the cell, which can jeopardize the entire experiment. ${ }^{30,31}$

Stability of the ZnSe-EDTA and ZnS:Mn-EDTA nanocrystal containing colloidal solutions was tested under various conditions. First, dispersibility and photostability of the EDTA capped nanocrystals in aqueous solutions were tested by storing the colloids, prepared by $c a .5 \mathrm{mg}$ corresponding nanocrystal solids dispersed in $10 \mathrm{~mL}$ water, in covered quartz cuvettes and standing under room light exposure for ca. 30 days. We did not observe any precipitation formed inside the cuvettes during that
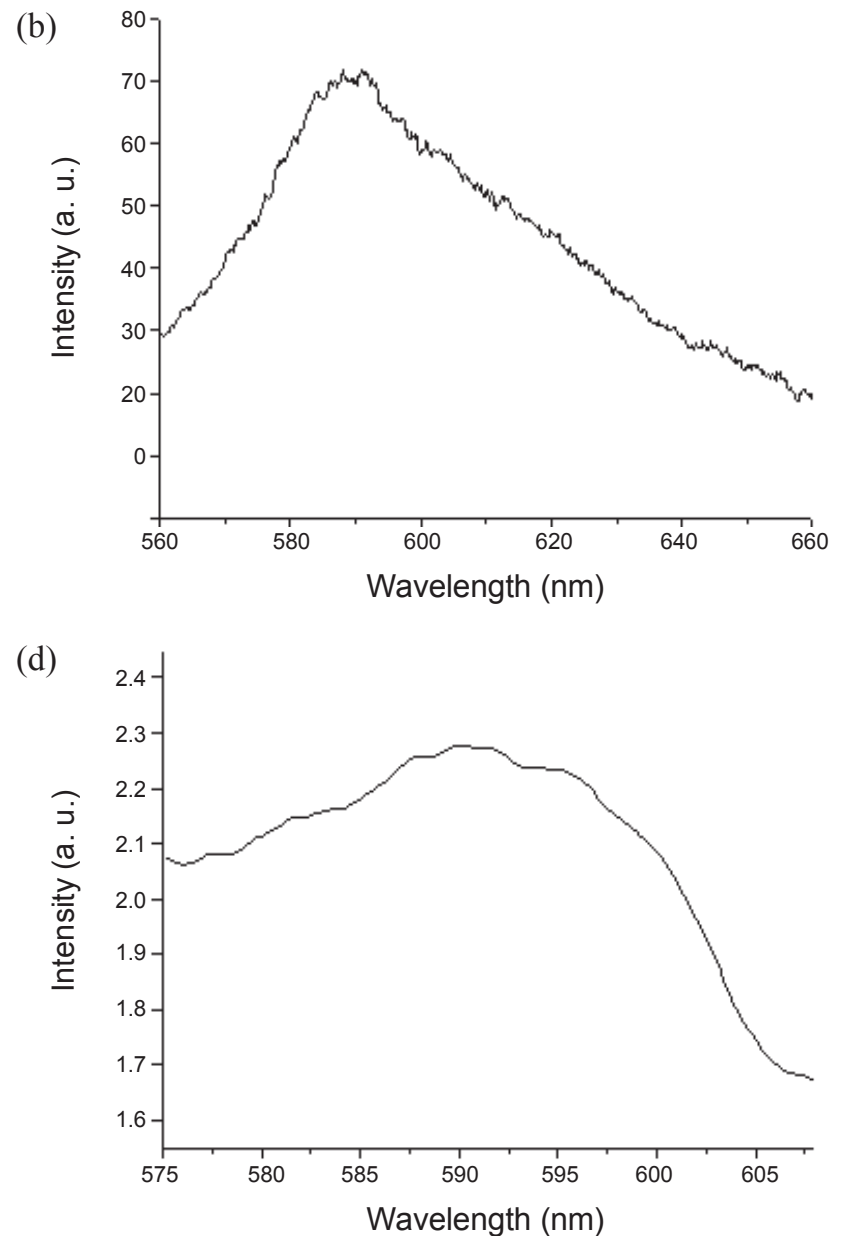

Figure 3. Room temperature solution PL spectra of: (a) ZnSe-EDTA and (b) ZnS:Mn-EDTA, and two-photon spectra of: (c) ZnSe-EDTA and (d) ZnS:Mn-EDTA. 
period, and further obtained PL spectra from the colloids showed that there were no significant changes in the intensity of the emission peaks compared to that for the Fig. 3(a) and (b), indicating that there were no significant changes in the EDTA capped nanocrystals, and they maintain fairly good dispersibility in water for at least 30 days. However, addition of ethanol resulted in formation of off-white cloudy solutions for both colloidal solutions. Second test was to estimate stability of the colloidal solutions against $\mathrm{pH}$ different environments. The nanocrystal containing colloidal solutions were split into two vials and 10 $\mathrm{mL}$ of $1.0 \mathrm{M} \mathrm{NaOH}$ and $10 \mathrm{~mL}$ of $1.0 \mathrm{M} \mathrm{HNO}_{3}$ stock solutions were added to the colloidal solutions containing ZnSe-EDTA and ZnS:Mn-EDTA nanocrystals. However, we did not observe any precipitation or color change in the resulting solutions; in addition, it did not cause any difference in PL spectra as well. Finally, third stability test for the colloids was performed by placing the ZnSe-EDTA and ZnS:Mn-EDTA colloidal solutions into a stainless steel pressure bomb and keeping the colloids under $120^{\circ} \mathrm{C}$ and 10 atm condition for 3 hours. We carried out this test because the procedure is very important for preparation of sterilized biological samples with the nanocrystals. The resulting solutions were clear with no precipitation and no color change; however, obtained PL spectra showed 20\% (ZnSeEDTA) and 15\% (ZnS:Mn-EDTA) decreased PL intensities compared to the corresponding original colloidal solutions, indicating that some of the capping molecules dissociate from the surface of the nanocrystals during the procedure to create additional surface defects on the ZnSe-EDTA and ZnS:MnEDTA nanocrystals under this severe condition.

Wide angle X-Ray diffraction patterns of the ZnSe-EDTA and $\mathrm{ZnS}: M n$-EDTA nanocrystals were obtained to confirm the formation of $\mathrm{ZnSe}$ and $\mathrm{ZnS}$ parent crystal lattices. The obtained peaks at (220), (311), (422) and (440) planes for ZnSe-EDTA, and (100), (002), (101), (110), (103) and (201) planes for the $\mathrm{ZnS}: M n-E D T A$ are identical to that for the corresponding hydrophobic nanocrystals, which indicates that there was no crystalline phase change during exchange the surface capping ligands, from TOPO to EDTA.

Finally, the capping ligand, EDTA, was also characterized by FT-IR spectroscopy. Wavenumbers of identifiable peaks in the FT-IR spectra are listed in Table 1. The peaks were assigned by a comparison with that of non-coordinated EDTA molecule, and some metal oxide bulk solid coordinated EDTA molecules reported in the literature. ${ }^{32}$ The carbonyl $(\mathrm{C}=\mathrm{O})$ group stretching peaks for ZnSe-EDTA (1637 $\mathrm{cm}^{-1}$ ) and ZnS:Mn-EDTA (1634 $\left.\mathrm{cm}^{-1}\right)$ were down shifted slightly from that of free-EDTA (1692 $\mathrm{cm}^{-1}$ ) molecule. In addition, the characteristic peaks of the antisymmetric and symmetric stretching modes of the COO group (for free EDTA; 1510 and $1396 \mathrm{~cm}^{-1}$ ) were also down shifted slightly for ZnSe-EDTA (1468 and $1394 \mathrm{~cm}^{-1}$ ) and ZnS:MnEDTA (1404 and $1320 \mathrm{~cm}^{-1}$ ). A similar pattern was observed for copper and iron oxide-coordinated EDTA molecules, in which the energy of the COO stretching modes were decreased by coordinating to a heavier $\mathrm{Cu}$ and $\mathrm{Fe}$ atoms than hydrogen. ${ }^{33}$ Therefore, the COO groups of ZnSe-EDTA and ZnS:Mn-EDTA are coordinated directly to the $\mathrm{Zn}$ metal ions of the nanocrystal surface. A similar phenomenon was observed in the FT-IR and Raman study with DFT calculations of the mercaptoacetic acid capped $\mathrm{ZnS}$ nanocrystals, in which the stretching peaks for $\mathrm{COO}$ and $\mathrm{SH}$ groups were down-shifted by directly coordinating to the $\mathrm{Zn}$ ion on the nanocrystal surface. ${ }^{34}$

\section{Conclusion}

Water-dispersible ZnSe and ZnS:Mn nanocrystals were synthesized by capping the originally hydrophobic surface of the nanocrystals with polar EDTA molecules. The optical properties of the colloidal nanocrystals obtained were measured by UV-visible and room temperature solution PL spectroscopy. The powders were characterized by XRD, HR-TEM, EDXS, and FT-IR spectroscopy. The solution PL spectra showed broad emission peaks at approximately 390 and $570 \mathrm{~nm}$, and with PL efficiencies of 4.4 and $2.4 \%$, respectively. In addition, the measured particle sizes of the amino acid-capped ZnS:Mn nanocrystals measured from the HR-TEM images were $5.4 \pm 1.2 \mathrm{~nm}$, and $4.7 \pm 0.8 \mathrm{~nm}$ for ZnSe-EDTA and ZnS:Mn-EDTA, respectively. One reason for synthesizing water-dispersible semiconductor nanocrystals was to introduce the nanocrystals to a bio-system as a biosensor material. Even though obtained ZnSeEDTA and ZnS:Mn-EDTA nanocrystals showed rather lower PL efficiencies than that for Cadmium based nanocrystals in aqueous solutions, we propose that those nanocrystals possess sufficient properties to be applied in bio-imaging or metal ion sensor area since we were able to obtain identifiable two-photon spectra as well as confirm colloidal stability in aqueous solvent for more than one month period. Some of the biological effects of the EDTA capped ZnSe and ZnS:Mn nanocrystals on living organisms are currently being tested.

Acknowledgments. This research was supported by the Graduate Research Assistantship of Dankook University in 2009-1 and 2009-2 (Jae-Woog Lee). In addition, this work was also supported by GRRC- Dankook 2009-B02 grant awarded from Gyonggi-Do.

\section{References}

1. Alivisatos, P. J. Phys. Chem. 1996, 100, 13226.

2. Goldman, E. R.; Balighian, H.; Mattoussi, M. K.; Mauro, J. M.; Tran, P. T.; Anderson, G. P. J. Am. Chem. Soc. 2002, 124, 6378.

3. Jaiswal, J. K.; Mattoussi, H.; Mauro, J. M.; Simon, S. M. Nature Biotechnol. 2002, 21, 47.

4. Milliron, D. J.; Alivisatos, A. P.; Pitois, C.; Edder, C.; Frechet, J. M. J. Adv. Mater. 2003, 15, 58

5. Pereiro, R.; Sanz-Medel, A.; Chang, W. H.; Parak, W. J. Mater. Chem. 2007, 17, 1343.

6. Hines, M. A.; Guyot-Sionnest, P. J. Phys. Chem. B 1998, 102, 3655.

7. Revaprasadu, N.; Malik, M. A.; O’Brien, P. J. Mater. Chem. 1998, 8,1885 .

8. Chestnoy, N.; Hull, R.; Brus, L. E. J. Chem. Phys. 1986, 85, 2237.

9. Song, K. K.; Lee, S. H. Curr. Appl. Phys. 2001, 1, 169.

10. Hwang, C. -S.; Cho, I. H. Bull. Kor. Chem. Soc. 2005, 26, 1776.

11. Hwang, J. M.; Oh, M. O.; Kim, I.; Lee, J. K.; Ha, C.-S. Curr. Appl. Phys. 2005, 5, 31 .

12. Hwang, C.-S.; Lee, N. R.; Kim, Y. A. and Park, Y. B. Bull. Kor. Chem. Soc. 2006, 27, 1809.

13. Lee, J. H.; Kim, Y. A.; Kim, K. M.; Huh, Y. D.; Hyun, J. W.; Kim, H. S.; Noh, S. J.; Hwang, C. -S. Bull. Kor. Chem. Soc. 2007, 28, 1091.

14. Mattousi, H.; Mauro, J. M.; Goldman, E. R.; Anderson, G. P.; 
Sundar, V. C.; Mikulec, F. V.; Bawendi, M. G. J. Am. Chem. Soc. 2000, 122, 12142.

15. Chan, W. C. W; Nie, S. Science 1998, 281, 2016; Alivisatos, P. Science 1996, 271, 933.

16. Gerion, D.; Pinaud, F.; Williams, S. C.; Parak, W. J.; Zanchet, D.; Weiss, S.; Alivisatos, A. P. J. Phys. Chem. B 2001, 195, 8861.

17. Chen, C. C.; Yet, C. P.; Wang, H. N.; Chao, C. Y. Langmuir 1999, 15,6845

18. Mitchell, G. P.; Mirkin, C. A.; Letsinger, R. L. J. Am. Chem. Soc. 1999, 121,8122 .

19. Jaiswal, J. K.; Mattoussi, H.; Mauro, J. M.; Simon, S. M. Nature Biotechnol. 2002, 21, 47.

20. Xin, R.; Ren, F.; Leng, Y. Mater. Des. 2010, 31, 1691.

21. Lee, T. M. J. Mater. Sci. 2006, 17, 15.

22. Xin, B. P.; We, J.; Guo, L. J. Inorg. Chem. 2009, 25, 774.

23. Melhuish, W. H. J. Phys. Chem. 1961, 65, 229.

24. Williams, A. T. R.; Winfield, S. A.; Miller, J. N. Analyst 1983,
$108,1067$.

25. Yi, G.; Sun, B.; Yang, F.; Chen, D. J. Mater. Chem. 2001, 11, 2928.

26. Kumbhokjar, N.; Mahamuni, S.; Leppert, V.; Risbud, S. H. Nanostruc. Mater. 1998, 10, 117.

27. Dong, B.; Cao, L.; Su, G.; Liu, W.; Zhai, H. J. Alloys and Comp. 2010, 429, 363.

28. Bhargava, R. N.; Gallagher, D.; Hong, X.; Nurmikko, A. Phys. Rev. Lett. 1994, 72, 416.

29. Breus, V. V.; Heys, C. D.; Nienhaus, G. U. J. Phys. Chem. 2007, 111, 18589.

30. Satzinger, V.; Schmidt, V.; Kuna, L. Micro. Opt. 2008, 17, 6992.

31. Zheng, L.; Zhang, Z. X. Prog. Biochem. Biophys. 2008, 35, 584.

32. Ryczkowski, J. Appl. Suf. Sci. 2005, 252, 813.

33. Nakamoto, K. Infrared and Raman Spectra of Inorganic and Coordination Compounds, 5th ed.; Wiley: 1997; Ch. 10.

34. Kim, J. E.; Hwang, C.-S.; Yoon, S. W. Bull. Kor. Chem. Soc. 2008, 29, 1247. 